\title{
"Go ask Alice, when she's 10-feet tall": Psychosocial correlates to lifetime LSD use among a national sample of US adults
}

\author{
ROBERT ANDREW YOCKEY ${ }^{1}$, KEITH A. KING ${ }^{1,2}$ and REBECCA A. VIDOUREK ${ }^{1,2}$ \\ ${ }^{1}$ Health Promotion and Education Program, University of Cincinnati, Cincinnati, OH, USA \\ ${ }^{2}$ Center for Prevention Science, University of Cincinnati, Cincinnati, OH, USA
}

(Received: February 16, 2019; accepted: May 12, 2019)

\begin{abstract}
Introduction: Once thought a rarely used drug, LSD use is steadily increasing among US adults. A greater understanding of social factors and psychological determinants leading to lifetime LSD use can assist health educators and professionals in treating this growing problem. This study analyzed psychosocial factors related to LSD use among a national sample of adults. Methods: A secondary data analysis of the 2017 National Survey on Drug Use and Health was performed. Results: Results from the final multivariate logistic regression revealed that those who were male, African American or Hispanic, used alcohol, ecstasy, marijuana, inhalants, cocaine, and cigarettes before the age of 21 years, thought about suicide, got a kick out of doing things risky, and tested oneself to do risky things were more likely to use LSD. Conclusions: This suggests that psychodynamic processes, for example, possible activation of emotional conflicts - can take place spontaneously - during ayahuasca intake in this particular setting. Some participants attributed symbolic meaning to the visionary content, which was more likely to take place in psychotherapeutically motivated clients. The specific setting influence as well as corresponding expectations of the participants in native wisdom could have considerable influence on experiences and interpretations, such as communication with entities as well as receiving personal teachings.
\end{abstract}

Keywords: LSD, psychosocial factors, substance use

\section{INTRODUCTION}

Colloquially known as "acid," "Golden Dragon," and "Yellow sunshine," lysergic acid diethylamide (LSD) use is a health problem in the USA. Part of a broader class of drugs known as psychedelics or hallucinogens, previous research estimated that a total of 30 million adults used psychedelics in their lifetime (Krebs \& Johansen, 2013). Data from the 2014 Global Drug Survey estimate the worldwide prevalence of LSD use to be $10.1 \%$. Repeated use of LSD and other hallucinogens has been linked to a myriad of health consequences including deficits in neurological processing, panic reactions, and substance use disorders (Abraham \& Aldridge, 1993; Liechti, 2017; Shalit, Rehm, \& Lev-Ran, 2019). Interestingly though, some have asserted the potential beneficial uses of hallucinogens in psychological therapies (e.g., reduced depression and enhanced mood) of hallucinogen use (Hendricks, Thorne, Clarke, Coombs, \& Johnson, 2015). Nevertheless, the overall efficacy of hallucinogenic therapy is greatly in need of case-control studies to verify such claims.

Specifically, with LSD, several pernicious consequences can occur as a result of use. LSD is known to affect neurocognition through the 5-HT-2A receptors, an important part of memory (Pagliaro \& Pagliaro, 2012). Moreover, recent research from Schmidt et al. (2018) found that acute LSD use impairs inhibitory performance and reduce neocortical activity. Other somatic toxicities, despite short-lived, can include anxiety, mood swings, visual illusions, and distortions of body image (Pagliaro \& Pagliaro, 2012).

The role of sex and ethnic differences remains unclear, particularly due to potential confounding variables, which combined psychedelic substances. When examining psychedelic substances, Palamar, Acosta, Sherman, Ompad, and Cleland (2016) found that males were more likely to use LSD and other substances than females. Examining LSD alone, the available literature suggests that no sex differences occur (Smart \& Fejer, 1969). Regarding ethnicity, Rickert, Siquiera, Dale, and Wiemann (2003) found a higher prevalence of LSD use among Caucasian females than females of other ethnic counterparts.

Several reasons for LSD use have been posited. Smart and Fejer (1969) interviewed LSD users and found that users perceived that LSD helped them to gain deeper insights to themselves, meditate, and become more focused in a variety of social situations. McGlothlin and Arnold (1971) found LSD users become more accepting and tolerant of themselves and others through taking LSD. Moreover, research by Barron, Lowinger, and Ebner (1970) found that sexual enhancement, escape, and sensory curiosity were alternative reasons offered for using LSD. Although much attention has

* Corresponding author: Robert Andrew Yockey, MS, Graduate Assistant; Health Promotion and Education Program, 2600 Clifton Avenue, Cincinnati, OH 45221-0068, USA; Phone/Fax: +1 513 442 9586; E-mail: yockeyra@mail.uc.edu

This is an open-access article distributed under the terms of the Creative Commons Attribution-NonCommercial 4.0 International License, which permits unrestricted use, distribution, and reproduction in any medium for non-commercial purposes, provided the original author and source are credited, a link to the CC License is provided, and changes - if any - are indicated. 
been devoted to such reasons, psychosocial risk factors need to be further explored. The purpose of this study was to examine specific risk factors related to lifetime LSD use among a national sample of adults.

A paucity of recent literature exists examining the factors associated with lifetime LSD use. Additional studies are warranted examining correlates with LSD use (Liechti, 2017). Moreover, most of the literature has combined psychedelic substances, instead of solely investigating LSD. A closer examination of specific psychosocial risk factors related to lifetime LSD use can assist health educators and professionals develop well-designed interventions to mitigate this growing problem. Additional research is needed from national, representative samples. Therefore, this study examined psychological variables and health risk factors related to lifetime and past-year LSD use among a national sample of adults in the USA. Specifically, this study sought out to address the following: (a) What percentage of adults report using LSD in their lifetime?, (b) Does lifetime LSD use differ based on sex and race?, (c) Does lifetime LSD use differ based on prior drug use before the age of 21 years?, (d) Does lifetime LSD use differ based on psychosocial correlates?

\section{METHODS}

\section{Instrument}

In this study, a secondary analysis of the 2017 National Survey on Drug Use and Health (NSDUH) was performed. The participants were delimited to individuals ranging 18-25 years old in the US and District of Columbia $(n=13,840)$. Recruitment of participants took place by the Research Triangle Institute (RTI). The RTI recruited participants using multistage area probability methods to select nonrepresentative samples of the US citizens. All adults who participated in the NSDUH self-identified as ages 18-25 years. This study was granted approval by the institutional review board at the University of Cincinnati. The US Federal Government designed the survey to provide nationwide LSD use behaviors among 18- to 25-year olds. There were three sections utilized from the NSDUH for this study: Lifetime LSD Use, Prior Substance Use, and Psychosocial Correlates.

\section{Lifetime LSD use}

To evaluate lifetime LSD use, a single question "Have you ever used LSD?" was asked. Responses were dichotomous in nature $(1=$ "yes" and $0=$ "no").

\section{Prior drug use}

To evaluate prior drug use, the NSDUH asked questions about drug use before the age of 18 years and before the age of 21 years. In this study, questions pertaining only to drug use before the age of 21 years were used. The following questions were utilized: "Have you ever used cigarettes before the age of 21 years?," "Have you ever used alcohol before the age of 21 years?," "Have you ever used cocaine before the age of 21 years?," "Have you ever used ecstasy before the age of 21 years?," "Have you ever used marijuana before the age of 21 years," and "Have you ever used inhalants before the age of 21 years?" Responses were dichotomous in nature $(1=$ "yes" and $0=$ "no").

\section{Psychosocial correlates}

To evaluate psychosocial correlates, the NSDUH incorporates 10 questions regarding a variety of psychological statuses and problems. Several questions were chosen from a team consensus. The following questions were asked: "Have you driven under the influence of alcohol within the past year?," "Do you get a real kick out of doing risky things?," "Do you like to test yourself by doing risky things?," "Do you wear a seatbelt when driving a car?," "Have you ever thought about suicide?," "Do you feel sad/empty, depressed most of the day or discouraged?," "Have you ever lost interest or pleasure in most things?," "Do you have sleep problems?," "Have you felt worthless nearly every day?," "How often have you felt nervous in past 30 days?," and "Do you have the inability to concentrate or make decisions?" Responses were dichotomous in nature $(1=$ "yes" and $0=$ "no" or $1=$ "all $/$ most of the time" and $0=$ "none of the time").

\section{Procedures}

The Substance Abuse and Mental Health Services Administration and the U.S. Department of Health and Human Services sponsor the NSDUH. To secure privacy and confidentiality, all interviews were conducted on the computer. No identifying information was revealed or asked on the survey. Both English and Spanish interviews were written. Both versions had the same content. Household participation was voluntary, and no other household could have replaced the requested participants. Upon completion of the interview, participants were compensated with $\$ 30$. The NSDUH has been shown to have good reliability and validity (Center for Behavioral Health Statistics and Quality, 2018).

\section{Data analysis}

All data were analyzed in Stata (v. 15.0, STATACorp., College Station, TX, USA) to account for the complex sampling design. Frequency distributions were used to calculate demographics and psychosocial characteristics of LSD users. A series of odds ratios (ORs) were calculated using weighted univariate logistic regression analyses. Significant variables were retained and a final multivariate logistic regression model was computed. To assess model fit, a non-significant Hosmer-Lemeshow test was utilized (Hosmer, Hosmer, Cessie, \& Lemeshow, 1997). In addition, the coefficient $R^{2}$ was used for model fit and to determine the variance accounted for between the independent and the dependent variables (Gordon, 2012).

\section{RESULTS}

\section{Demographics}

A total of 13,840 adults aging $18-25$ years participated in the survey by completing the 2017 NSDUH (Table 1). A total of $8.8 \%$ of adults $(n=1,239)$ reported lifetime LSD use. 
Table 1. Demographics

\begin{tabular}{|c|c|c|c|}
\hline \multirow[b]{2}{*}{ Variable } & \multicolumn{3}{|c|}{ Lifetime LSD use } \\
\hline & $N(\%)$ & OR & {$[95 \% \mathrm{CI}]$} \\
\hline \multicolumn{4}{|l|}{ Sex } \\
\hline Females $^{\mathrm{a}}$ & $7,136(51.6)$ & 1.00 & \\
\hline Males & $6,704(48.4)$ & $0.493 * * *$ & {$[0.437,0.557]$} \\
\hline \multicolumn{4}{|l|}{ Race } \\
\hline White $^{\mathrm{a}}$ & $7,496(54.2)$ & 1.00 & \\
\hline African American/Black & $1,890(13.7)$ & $5.14 * * *$ & {$[3.82,6.93]$} \\
\hline Native Americans & $227(1.6)$ & 1.523 & {$[0.936,2.48]$} \\
\hline Native HI/Pacific Islanders & $68(0.49)$ & 2.841 & {$[0.891,9.06]$} \\
\hline Asians & $678(4.89)$ & $3.58 * * *$ & {$[2.37,5.41]$} \\
\hline Multiracial & $614(4.4)$ & 0.942 & {$[0.733,5.41]$} \\
\hline Hispanics & $2,866(20.7)$ & $1.72 * * *$ & {$[1.47,2.02]$} \\
\hline \multicolumn{4}{|l|}{ Self-reported health status } \\
\hline Excellent/very good/good ${ }^{\mathrm{a}}$ & $12,854(92.9)$ & 1.00 & \\
\hline Fair/poor & $986(7.1)$ & $1.41 * * *$ & {$[1.15,1.72]$} \\
\hline \multicolumn{4}{|l|}{ County } \\
\hline Non-metro ${ }^{a}$ & 2,631 (19.0) & 1.00 & \\
\hline Large/small metro & $11,209(81.0)$ & 1.19 & {$[1.02,1.39]$} \\
\hline
\end{tabular}

Note. $N=13,840$; percent refers to valid percent; missing values excluded. LSD: lysergic acid diethylamide; OR: odds ratio; CI: confidence interval.

${ }^{\mathrm{a}}$ Reference category.

$* * * p<.001$.

Table 2. Prior drug use to lifetime LSD use

\begin{tabular}{|c|c|c|c|c|}
\hline \multirow[b]{2}{*}{ Variable } & \multicolumn{4}{|c|}{ Lifetime LSD use } \\
\hline & No lifetime LSD use $[n(\%)]$ & Lifetime LSD use $[n(\%)]$ & OR & {$[95 \% \mathrm{CI}]$} \\
\hline \multicolumn{5}{|c|}{ First used alcohol before age $21 ?$} \\
\hline $\mathrm{No}^{\mathrm{a}}$ & $3,990(99.4)$ & $24(0.6)$ & 1.00 & \\
\hline Yes & $8,611(87.6)$ & $1,215(12.4)$ & $23.5^{* * *}$ & {$[15.6,35.2]$} \\
\hline \multicolumn{5}{|c|}{ First used cigarettes before age $21 ?$} \\
\hline $\mathrm{No}^{\mathrm{a}}$ & $7,025(98.3)$ & $118(1.7)$ & 1.00 & \\
\hline Yes & $5,576(83.3)$ & $1,121(16.7)$ & $12.0 * * *$ & {$[9.87,14.5]$} \\
\hline \multicolumn{5}{|c|}{ First used cocaine before age $21 ?$} \\
\hline $\mathrm{No}^{\mathrm{a}}$ & $12,031(94.9)$ & $646(5.1)$ & 1.00 & \\
\hline Yes & $570(49.0)$ & $593(51.0)$ & $19.4^{* * *}$ & {$[16.9,22.3]$} \\
\hline \multicolumn{5}{|c|}{ First used ecstasy before age $21 ?$} \\
\hline $\mathrm{No}^{\mathrm{a}}$ & $12,042(95.8)$ & $522(4.2)$ & 1.00 & \\
\hline Yes & $559(43.8)$ & $717(56.2)$ & $29.6 * * *$ & {$[25.7,34.1]$} \\
\hline \multicolumn{5}{|c|}{ First used inhalants before age $21 ?$} \\
\hline $\mathrm{No}^{\mathrm{a}}$ & $11,764(93.1)$ & $875(6.9)$ & 1.00 & \\
\hline Yes & $837(69.7)$ & $364(30.3)$ & $5.85 * * *$ & {$[5.08,6.73]$} \\
\hline \multicolumn{5}{|c|}{ First use marijuana before age $21 ?$} \\
\hline $\mathrm{No}^{\mathrm{a}}$ & $6,999(99.6)$ & $29(0.4)$ & 1.00 & \\
\hline Yes & $5,602(82.2)$ & $1,210(17.8)$ & $52.1 * * *$ & {$[36.0,75.5]$} \\
\hline \multicolumn{5}{|c|}{ Drove under the influence of alcohol within the past year? } \\
\hline $\mathrm{No}^{\mathrm{a}}$ & $11,383(92.6)$ & $914(7.4)$ & 1.00 & \\
\hline Yes & $1,114(77.6)$ & $322(22.4)$ & $3.60 * * *$ & {$[3.13,4.15]$} \\
\hline
\end{tabular}

Note. $N=13,840$; percent refers to valid percent; missing values excluded. LSD: lysergic acid diethylamide; OR: odds ratio; CI: confidence interval.

${ }^{\mathrm{a}}$ Reference category.

$* * * p<.001$

\section{Lifetime LSD use based on other substance use}

Males were more likely to report lifetime LSD use $\left(\chi^{2}=134.7, \mathrm{OR}=0.493, p<.001\right)$ than females. The results indicated that lifetime LSD use was associated with other use of illicit substances (Table 2). Compared to their counterparts, adults who reported using substances before the age of 21 years were 23.5 times more likely, who 
reported using cigarettes were 12.0 times more likely, who reported using cocaine before 21 were 19.4 times more likely, who reported using ecstasy before 21 were 29.6 times more likely, who reported inhalant use before 21 were 5.85 times more likely, and who reported marijuana before 21 were 52.1 times more likely to have reported lifetime LSD use (Table 2).

\section{Psychosocial correlates}

The results indicated that psychosocial correlates were related to lifetime LSD use (Table 3). Compared to their counterparts, adults who reported getting a real kick out of doing something dangerous or risky $(\mathrm{OR}=3.50, p<.001)$, liked to test themselves by doing risky things $(\mathrm{OR}=3.13$, $p<.001)$, never/seldom wearing a seatbelt $(\mathrm{OR}=1.73$, $p<.001)$, thought about suicide ( $\mathrm{OR}=2.46, p<.001)$, were $\mathrm{sad} /$ empty/depressed most of day or discouraged $(\mathrm{OR}=2.13$, $p<.001)$, lost interest or pleasure in most things ( $\mathrm{OR}=2.14$, $p<.001)$, reported sleep problems $(\mathrm{OR}=2.08, p<.001)$, felt worthless $(\mathrm{OR}=2.15, p<.001)$, felt nervous all $/$ most of the time in the past 30 days $(\mathrm{OR}=1.58, p<.001)$, and had the inability to concentrate or make decisions $(\mathrm{OR}=2.11, p<.001)$ were more likely to have reported lifetime LSD use.

\section{Multivariate logistic regression}

As mentioned above, the univariate unadjusted ORs revealed that sex, race, first use of alcohol, marijuana, ecstasy, cocaine, inhalants, and cigarettes before the age of 21 years, getting a kick out of doing something dangerous/ risky, testing yourself by doing risky things, not wearing a seat belt, thinking about suicide, sad/empty depressed, lost pleasure in doing things enjoyable, sleep problems, feeling worthless, feeling nervous, and inability to concentrate/ make decisions were all significant predictors. These predictors were then put into a final multivariate logistic regression model to determine significant predictors of lifetime LSD use (Table 4). The model predicted lifetime LSD significantly (omnibus $\chi^{2}=3,493.062, d f=23$, $p<.001$ ) and predicted $22.4 \%-49.4 \%$ of the variance in

Table 3. Psychosocial correlates to lifetime LSD use

\begin{tabular}{|c|c|c|c|c|}
\hline \multirow[b]{2}{*}{ Variable } & \multicolumn{4}{|c|}{ Lifetime LSD use } \\
\hline & No lifetime LSD use $[n(\%)]$ & Lifetime LSD use $[n(\%)]$ & OR & {$[95 \% \mathrm{CI}]$} \\
\hline \multicolumn{5}{|c|}{ Get a real kick out of doing something dangerous/risky? } \\
\hline $\mathrm{No}^{\mathrm{a}}$ & $9,159(94.5)$ & $535(5.5)$ & 1.00 & \\
\hline Yes & $3,442(83.0)$ & $704(17.0)$ & $3.50 * * *$ & {$[3.11,3.94]$} \\
\hline \multicolumn{5}{|c|}{ Like to test yourself by doing risky things? } \\
\hline $\mathrm{No}^{\mathrm{a}}$ & $9,812(93.7)$ & $656(6.3)$ & 1.00 & \\
\hline Yes & $2,789(82.7)$ & $583(17.3)$ & $3.13 * * *$ & {$[2.78,3.52]$} \\
\hline \multicolumn{5}{|c|}{ How often do you wear a seatbelt when you drive a car? } \\
\hline Always/sometimes ${ }^{\mathrm{a}}$ & $11,860(91.4)$ & $1,118(8.6)$ & 1.00 & \\
\hline Never/seldom & $741(86.0)$ & $121(14.0)$ & $1.73 * * *$ & {$[1.42,2.12]$} \\
\hline \multicolumn{5}{|l|}{ Thought about suicide? } \\
\hline $\mathrm{No}^{\mathrm{a}}$ & $11,426(92.0)$ & $989(8.0)$ & 1.00 & \\
\hline Yes & $1,175(82.5)$ & $250(17.5)$ & $2.46^{* * *}$ & {$[2.11,2.86]$} \\
\hline \multicolumn{5}{|c|}{ Sad/empty/depressed most of day or discouraged? } \\
\hline $\mathrm{No}^{\mathrm{a}}$ & $10,320(92.4)$ & $843(7.6)$ & 1.00 & \\
\hline Yes & $2,281(85.2)$ & $396(14.8)$ & $2.13 * * *$ & {$[1.87,2.42]$} \\
\hline \multicolumn{5}{|c|}{ Lost interest or pleasure in most things? } \\
\hline $\mathrm{No}^{\mathrm{a}}$ & $10,375(92.4)$ & $849(7.6)$ & 1.00 & \\
\hline Yes & $2,226(85.1)$ & $390(14.9)$ & $2.14 * * *$ & {$[1.88,2.43]$} \\
\hline \multicolumn{5}{|l|}{ Sleep problems? } \\
\hline $\mathrm{No}^{\mathrm{a}}$ & $10,307(92.4)$ & $847(7.6)$ & 1.00 & \\
\hline Yes & $2,294(85.4)$ & $392(14.6)$ & $2.08 * * *$ & {$[1.83,2.36]$} \\
\hline \multicolumn{5}{|l|}{ Felt worthless nearly every day? } \\
\hline $\mathrm{No}^{\mathrm{a}}$ & $11,068(92.1)$ & $955(7.9)$ & 1.00 & \\
\hline Yes & $1,533(84.4)$ & $284(15.6)$ & $2.15^{* * *}$ & {$[1.86,2.48]$} \\
\hline \multicolumn{5}{|c|}{ How often felt nervous past 30 days? } \\
\hline Some/little/none of the time ${ }^{a}$ & $10,390(91.6)$ & $998(8.4)$ & 1.00 & \\
\hline All/most of the time & $1,671(87.4)$ & $241(12.6)$ & $1.58 * * *$ & {$[1.36,1.84]$} \\
\hline \multicolumn{5}{|c|}{ Inability to concentrate or make decisions? } \\
\hline No & $10,562(92.3)$ & $881(7.7)$ & 1.00 & \\
\hline $\mathrm{Yes}^{\mathrm{a}}$ & $2,039(85.1)$ & $358(14.9)$ & $2.11^{* * *}$ & {$[1.84,2.40]$} \\
\hline
\end{tabular}

Note. $N=13,840$; percent refers to valid percent; missing values excluded. LSD: lysergic acid diethylamide; OR: odds ratio; CI: confidence interval.

${ }^{\mathrm{a}}$ Reference category.

$* * * p<.001$. 
Table 4. Multivariate logistic regression for lifetime LSD use

\begin{tabular}{lrrrcc}
\hline Item & \multicolumn{1}{c}{$B$} & \multicolumn{1}{c}{$S E$} & Wald & Exp $b$ & {$[95 \% \mathrm{CI}]$} \\
\hline Sex (male) & -0.670 & 0.081 & 67.954 & 0.512 & {$[0.436,0.600]^{* * *}$} \\
Race (Black/African Americans) & 0.047 & 0.032 & 2.012 & 2.104 & {$[1.974,2.896]^{* * *}$} \\
Race (Hispanic) & 0.068 & 0.140 & 0.240 & 1.071 & {$[0.814,1.409]^{* * *}$} \\
Self-reported health status (fair/poor) & 0.047 & 0.135 & 0.122 & 1.048 & {$[0.804,1.366]$} \\
First used alcohol before age 21 (yes) & 0.766 & 0.230 & 11.074 & 2.151 & {$[1.370,3.376]^{* * *}$} \\
First used cigarettes before age 21 (yes) & 0.792 & 0.113 & 49.393 & 2.207 & {$[1.770,2.752]^{* * *}$} \\
First used cocaine before age 21 (yes) & 0.890 & 0.093 & 91.469 & 2.434 & {$[2.029,2.923]^{* * *}$} \\
First used ecstasy before age 21 (yes) & 2.002 & 0.087 & 529.729 & 7.401 & {$[6.241,8.776]^{* * *}$} \\
First used marijuana before age 21 (yes) & 2.363 & 0.203 & 135.004 & 10.619 & {$[7.129,15.819]^{* * *}$} \\
First used inhalants before age 21 (yes) & 0.414 & 0.097 & 18.280 & 1.513 & {$[1.251,1.828]^{* * *}$} \\
Drove under the influence of alcohol within the past year (yes) & 0.015 & 0.096 & 0.024 & 1.015 & {$[0.841,1.225]$} \\
Get a real kick out of doing risky things (yes) & 0.359 & 0.095 & 13.830 & 1.431 & {$[1.181,1.724]^{* * *}$} \\
Like to test yourself by doing risky things (yes) & 0.217 & 0.096 & 5.057 & 1.253 & {$[1.028,1.513]^{* *}$} \\
Wear a seatbelt when you drive a car (no) & -0.206 & 0.136 & 2.310 & 0.813 & {$[0.623,1.062]$} \\
Thought about suicide (yes) & 0.323 & 0.113 & 8.187 & 1.381 & {$[1.107,1.722]^{* * *}$} \\
Sad/empty/depressed most of day or discouraged (yes) & 0.298 & 0.241 & 1.531 & 1.347 & {$[0.840,2.161]$} \\
Lost interest or pleasure in most things (yes) & -0.008 & 0.227 & 0.001 & 1.008 & {$[0.647,1.571]$} \\
Sleep problems (yes) & -0.063 & 0.253 & 0.062 & 0.939 & {$[0.572,1.543]$} \\
Felt worthless nearly every day (yes) & -0.041 & 0.159 & 0.066 & 0.960 & {$[0.703,1.311]$} \\
How often felt nervous in past 30 days (all/most of the time) & -0.006 & 0.107 & 0.003 & 0.994 & {$[0.807,1.226]$} \\
Inability to concentrate or make decisions (yes) & 0.108 & 0.203 & 0.283 & 0.960 & {$[0.703,1.661]$} \\
\hline
\end{tabular}

Note. $N=13,840$; percent refers to valid percent; missing values excluded. The model significantly predicted lifetime LSD use (omnibus $\left.\chi^{2}=3,502.800, d f=24, p<.001\right)$ and accounted for $22.4 \%-49.4 \%$ of the variance in lifetime LSD use. SE: standard error; LSD: lysergic acid diethylamide;

OR: odds ratio; CI: confidence interval.

$* * * p<.001$

lifetime LSD use. In addition, the model fit well (HosmerLemeshow test: $\chi^{2}=5.079, d f=8, p=.680$ ). Statistically significant predictors were sex, race, first use of alcohol, marijuana, ecstasy, cocaine, inhalants, and cigarettes before the age of 21 years, getting a kick out of doing something dangerous/risky, liking to test yourself by doing risky things, and thinking about suicide (Table 3). Those at highest risk for lifetime use of LSD use were male $(\mathrm{OR}=0.512, p<.001)$, Black/African American $(\mathrm{OR}=$ $2.104, p<.001)$, Hispanic $(\mathrm{OR}=1.071, p<.001)$ used alcohol before age $21(\mathrm{OR}=2.151, p<.001)$, used cigarettes before age $21(\mathrm{OR}=2.207, p<.001)$, used cocaine before age $21(\mathrm{OR}=2.434, p<.001)$, used ecstasy before age $21(\mathrm{OR}=7.401, p<.001)$, used marijuana before age 21 $(\mathrm{OR}=10.619, p<.001)$, used inhalants before the age of 21 years $(\mathrm{OR}=1.513, p<.001)$, got a real kick out of doing risky things $(\mathrm{OR}=1.431, p<.001)$, liked to test themselves by doing risky things $(\mathrm{OR}=1.253, p<.001)$, and had thoughts about suicide $(\mathrm{OR}=1.381, p<.001)$.

\section{DISCUSSION}

LSD use continues to be a health concern in the USA. Delineation of risk factors can assist health educators and professionals in developing certain interventions designed to mitigate this problem. This study analyzed psychosocial risk factors for lifetime LSD use. The results indicated that being male, Hispanic or African American, using marijuana, cigarettes, alcohol, ecstasy, inhalants, and cocaine before the age of 21 , getting a real kick out of doing risky things, testing yourself by doing risky things, and thinking about suicide were all significant risk factors for lifetime LSD use.

Those who reported using alcohol before the age of 21 years were 23.5 times more likely to report lifetime LSD use. Prior research (Kandel, Yamaguchi, \& Chen, 1992) found that alcohol preceded other illicit substance use, in particular marijuana and psychoactive drugs, among young adults. In addition, Yu and Williford (1992) found that alcohol use predicted future use of marijuana, cigarettes, and other drugs. Future research should examine the link and trajectory between prior alcohol use and LSD use.

Those who reported using cigarettes before the age of 21 years were at elevated risk of reporting lifetime LSD use. Exposure to cigarettes early in life may predispose one to try other substances for a more intense physical and mental effect. Weinberger and Sofuoglu (2009) found a strong link between cigarette smoking and stimulant addiction in both animals and humans, suggesting a more profound influence of tobacco on the development of other substances. Health professionals should target cigarette smoking at an early age. Specifically, the use of well-designed media campaigns may lower the use of the cigarettes and possibly prevent the use of LSD in the future among adults.

The use of marijuana before the age of 21 years increased the risk of lifetime LSD use. Wilcox, Wagner, and Anthony (2002) found from a national database that youths who used marijuana had higher odds of using hallucinogens than nonusers of marijuana. Secades-Villa, Garcia-Rodriguez, Jin, Wang, and Blanco (2015) found that among adults, those who used marijuana have progressed to other illicit drugs at 
a faster rate. Marijuana has long been criticized as a "gateway" drug, as both developmental and biological models posit (Pagliaro \& Pagliaro, 2012). By using marijuana, users can gradually move up to more hardcore drugs. Significantly, it is critical to consider the link between LSD/ hallucinogen use and marijuana use when developing health interventions and policies.

Illicit substances, such as cocaine, inhalants, and ecstasy, used before the age of 21 years were all linked to an elevated risk of LSD use. The use of multiple substances with LSD may be cross-cultural in nature. Recent research from Matthews et al. (2017) suggests that cocaine and LSD were used primarily among psychostimulant users. A possible explanation for this novel relationship is that one of these drugs may enhance the effects of LSD, suggesting further use of LSD.

Sensation and risk seeking have longed been linked to substance use and other illicit drug use (Pagliaro \& Pagliaro, 2012). The results from this study corroborated this fact, with adults who liked to test themselves or engaged in risky behaviors more likely to use LSD. Perhaps by performing risky behaviors, one increases their sense of superiority, thus increasing their risk to try more perilous behaviors. Health professionals should examine the aspects to risk seeking more closely when developing health interventions.

This study found that those who reported thinking about suicide were 1.38 times more likely to use LSD. This finding is unique, as previous research has found no relationship between psychedelic use and suicidal behavior (Johansen \& Krebs, 2015). Hendricks et al. (2015) analyzed national data and found decreased suicidal thinking among psychedelic users. Moreover, a therapeutic benefit was found, as Hendricks et al. posited that psychedelic drugs may hold promising aspects in the prevention of suicidal behaviors. LSD has been used for "escape," and perhaps those who use LSD may feel that it helps them to escape thoughts of suicide. The relationship between psychedelic use, particularly LSD, and suicidal behavior remains nebulous and future research is warranted. However, the potential perceived beneficial effects should be considered taking into account the plethora of harmful effects the LSD possesses (Abraham \& Aldridge, 1993; Liechti, 2017).

\section{Limitations}

Several limitations of this study exist. First, the NSDUH is cross-sectional in nature, so no causal relationships can be established. The use of self-reported data increases the risk of under/overreporting. We limited the data to 18- to 25 -year olds, so generalizability to other populations is limited. In addition, some participants may have responded in a socially desirable manner. Finally, the NSDUH does not assess why individuals use hallucinogenic drugs. Future research is essential to identify specific reasons individuals use LSD.

\section{CONCLUSIONS}

LSD use continues to be a health concern among adults. This study identified potential risk mechanisms associated with lifetime LSD use. Those at highest risk of lifetime LSD use were male, African American or Hispanic, first used marijuana, inhalants, ecstasy, cigarettes, alcohol, and cocaine before the age of 21 years, thought about suicide, likes to test themselves by doing risky things, and got a "kick" out of doing risky things. The findings from this study could help health professionals develop prevention initiatives and programs aimed at alleviating LSD use. Messages framed at accurately delineating the harms and potential consequences of LSD use are warranted.

Acknowledgements: No financial support supported this study. RAY would like to thank KAK and RAV for their guidance and support for the study design and analysis. KAK assisted with writing and editing the manuscript and RAV contributed to manuscript edits.

Conflict of interest: The authors declare no conflict of interest.

\section{REFERENCES}

Abraham, H. D., \& Aldridge, A. M. (1993). Adverse consequences of lysergic acid diethylamide. Addiction, 88(10), 1327-1334. doi:10.1111/j.1360-0443.1993.tb02018.x

Barron, S. P., Lowinger, P., \& Ebner, E. (1970). A clinical examination of chronic LSD use in the community. Comprehensive Psychiatry, 11(1), 69-79. doi:10.1016/0010-440X(70) 90206-3

Center for Behavioral Health Statistics and Quality. (2018). 2017 national survey on drug use and health: Methodological summary and definitions. Rockville, MD: Substance Abuse and Mental Health Services Administration.

Gordon, R. A. (2012). Applied statistics for the social and health sciences. New York, NY: Routledge.

Hendricks, P. S., Thorne, C. B., Clarke, C. B., Coombs, D. W., \& Johnson, M. W. (2015). Classic psychedelic use is associated with reduced psychological distress and suicidality in the United States population. Journal of Psychopharmacology, 29(3), 280-288. doi:10.1177/0269881114565653

Hosmer, D. W., Hosmer, T., Cessie, S. L., \& Lemeshow, S. (1997). A comparison of goodness of-fit tests for the logistic regression model. Statistics in Medicine, 16(9), 965-980. doi:10.1002/(SICI) 1097-0258(19970515)16:9<965::AID-SIM509>3.0.CO;2-O

Johansen, P. O., \& Krebs, T. S. (2015). Psychedelics not linked to mental health problems or suicidal behavior: A population study. Journal of Psychopharmacology, 29(3), 270-279. doi:10.1177/0269881114568039

Kandel, D. B., Yamaguchi, K., \& Chen, K. (1992). Stages of progression in drug involvement from adolescence to adulthood: Further evidence for the gateway theory. Journal of Studies on Alcohol, 53(5), 447-457. doi:10.15288/jsa.1992.53.447

Krebs, T. S., \& Johansen, P. O. (2013). Over 30 million psychedelic users in the United States. F1000 Research, 2, 98-103. doi:10.12688/f1000research.2-98.v1

Liechti, M. E. (2017). Modern clinical research on LSD. Neuropsychopharmacology, 42(11), 2114-2127. doi:10.1038/npp.2017.86 
Matthews, A., Sutherland, R., Peacock, A., Buskirk, J. V., Whittaker, E., Burns, L., \& Bruno, R. (2017). I like the old stuff better than the new stuff? Subjective experiences of new psychoactive substances. International Journal of Drug Policy, 40, 44-49. doi:10.1016/j.drugpo.2016.11.004

McGlothlin, W. H., \& Arnold, D. O. (1971). LSD revisited: A ten-year follow-up of medicinal LSD use. Archives of General Psychiatry, 24(1), 35-49. doi:10.1001/archpsyc.1971.01750070037005

Pagliaro, L. A., \& Pagliaro, L. A. (2012). Handbook of child and adolescent drug and substance abuse: Pharmacological, developmental, and clinical considerations. Hoboken, NJ: John Wiley \& Sons, Inc.

Palamar, J. J., Acosta, P., Sherman, S., Ompad, D. C., \& Cleland, C. M. (2016). Self-reported use of novel psychoactive substances among attendees of electronic music dance venues. The American Journal of Drug and Alcohol Abuse, 42(6), 624-632. doi:10.1080/00952990.2016.1181179

Rickert, V. I., Siquiera, L. M., Dale, T., \& Wiemann, C. M. (2003). Prevalence and risk factors for LSD use among young women. Journal of Pediatric and Adolescent Gynecology, 16(2), 67-75. doi:10.1016/S1083-3188(03)00012-3

Schmidt, A., Müller, F., Lenz, C., Dolder, P. C., Schmid, Y., Zanchi, D., Lang, U. E., Liechti, M. E., \& Bordwardt, S. (2018). Acute LSD effects on response inhibition neural networks. Psychological Medicine, 48(9), 1464-1473. doi:10. 1017/S0033291717002914
Secades-Villa, R., Garcia-Rodriguez, O., Jin, C. J., Wang, S., \& Blanco, C. (2015). Probability and predictors of the cannabis gateway effect: A national study. International Journal of Drug Policy, 26(2), 135-142. doi:10.1016/j.drugpo.2014.07.011

Shalit, N., Rehm, J., \& Lev-Ran, S. (2019). Epidemiology of hallucinogen use in the U.S.: Results from the National Epidemiologic Survey on Alcohol and Related Conditions III. Addictive Behaviors, 89, 35-43. doi:10.1016/j.addbeh. 2018.09.020

Smart, R. G., \& Fejer, D. (1969). Illicit LSD users: Their social backgrounds, drug use, and psychopathology. Journal of Health and Social Behavior, 10(4), 297-308. doi:10.2307/ 2948437

Weinberger, A. H., \& Sofuoglu, M. (2009). The impact of cigarette smoking on stimulant addiction. The American Journal of Drug and Alcohol Abuse, 35(1), 12-17. doi:10.1080/0095299 0802326280

Wilcox, H. C., Wagner, F. A., \& Anthony, J. C. (2002). Exposure opportunity as a mechanism linking youth marijuana use to hallucinogen use. Drug and Alcohol Dependence, 66(2), 127-135. doi:10.1016/S0376-8716(01)00191-0

Yu, J., \& Williford, W. R. (1992). The age of alcohol onset and alcohol, cigarette, and marijuana use patterns: An analysis of drug use progression of young adults in New York state. International Journal of the Addictions, 27(11), 1313-1323. doi:10.3109/10826089209047353 